\title{
Theoretical and Numerical Investigations of Parametric Transfer via Difference Frequency Generation for Indirect Mid-Infrared Pulse Shaping
}

\author{
Jerry Prawiharjo, Hazel S. S. Hung, David C. Hanna, David P. Shepherd \\ Optoelectronics Research Centre, University of Southampton, Southampton SO17 1BJ, \\ United Kingdom
}

\begin{abstract}
We theoretically and numerically investigate indirect mid-infrared pulse shaping via parametric transfer, specifically difference-frequency generation. We define a quantitative measure for the fidelity of parametric transfer, and investigate the effect of material dispersion and process nonlinearity on the parametric transfer. We show that a good fidelity transfer of a broadbandwidth pulse can be efficiently achieved with a reasonable wavelength tunability, by careful design of the experimental configuration. (c) 2006 Optical Society of America

OCIS codes: 190.4410, 190.4970, 320.5540, 320.7110.
\end{abstract}

\section{Introduction}

Ultrashort optical pulses in the mid-infrared (MIR) spectral regime have become powerful tools in both fundamental and applied research. The combination of their quantum energy and timescale not only allows insight into the ultrafast dynamics of various materials, ${ }^{1-3}$ but is also useful for controlling the outcome of quantum phenomena. ${ }^{4-7}$ Crucial to these experiments is the ability to tailor the intensity and phase of these MIR pulses precisely. Furthermore, electronically programmable methods are often required for versatile pulseshaping, especially in coherent control experiments where closed-loop learning techniques ${ }^{4}$ are necessary.

Direct electronically programmable pulse-shaping techniques are now routinely used for pulses in the visible and near-infrared (NIR) region using liquid-crystal modulators (LCMs), ${ }^{8}$ deformable mirrors, ${ }^{9}$ and acousto-optic modulators (AOMs). ${ }^{10}$ Their implementation in the MIR spectral region is, however, nontrivial. For example, LCMs are opaque in this wavelength range, while the deformable mirrors require a large deflection to achieve a significant phase modulation. Although a direct programmable MIR pulse shaping using a Germanium AOM 
has been successfully demonstrated recently, ${ }^{11}$ its limited radio frequency bandwidth limits its modulation resolution and its applicability to a pulse train with a high repetition rate.

An alternative approach to this problem is through indirect pulse shaping, that is, to shape ultrashort optical pulses in the visible or NIR and to transfer them to the MIR region via a nonlinear optical process. The first successful attempt in this approach was achieved via difference-frequency generation (DFG) between the spectral wings of a broadband pulse from a titanium sapphire laser. ${ }^{12,13}$ This method, however, is limited in terms of spectral tunability and output energy. Better results can be achieved by performing DFG between two pulses, a shaped pulse and another one without complex structure, at different wavelengths. ${ }^{14-17}$

To the best of our knowledge, previous works reporting experimental demonstrations of this parametric transfer provided limited discussion on the factors which can limit its performance. In this paper, we theoretically and numerically investigate parametric transfer via DFG. We reexamine the convolution and provide quantitative measures of the fidelity of parametric transfer using spectrograms. We also analyze the effects of material dispersion, which is manifest in temporal walk-off and group velocity dispersion, and of process nonlinearity, which is manifest in pump depletion and signal amplification. Our results confirm that one can efficiently transfer broad bandwidth pulses with good fidelity and reasonable tuning ranges.

This paper is organized as follows. In Section 2, we present the indirect pulse shaping technique via DFG through a simple convolution, define the quantitative measure of parametric transfer, and introduce the shape of the pump pulse we will analyze in this work. We then continue with the theoretical analysis of the DFG in the presence of material dispersion, specifically temporal walk-off, in Section 3, followed by extensive numerical analysis in Section 4, which also includes process nonlinearity and group velocity dispersion. These results are, in principle, applicable to any second-order nonlinear material. In Section 5, we specifically address the parametric transfer in periodically-poled lithium niobate (PPLN), which has been widely used for frequency conversion. We then provide discussions of our results in Section 6, including their implications for parametric transfer in optical parametric oscillators (OPOs).

\section{Indirect Pulse Shaping via Difference-Frequency Generation}

We investigate an indirect pulse shaping technique via parametric transfer as illustrated schematically in Fig. 1. A shaped ultrashort pulse, henceforth referred to as the pump pulse, with a carrier frequency $\omega_{p}$ in the NIR regime, is mixed with a signal pulse, with a carrier frequency $\omega_{s}<\omega_{p}$, also in the NIR regime, via DFG in a second-order nonlinear material to generate a shaped idler pulse with a carrier frequency $\omega_{i}=\omega_{p}-\omega_{s}$ in the MIR regime. This scheme has been experimentally demonstrated in Ref. 17, and differs from others, ${ }^{14-16}$ 


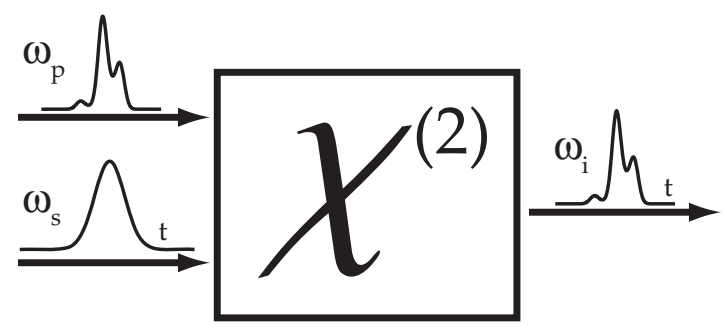

Fig. 1. Illustration of indirect pulse shaping via parametric transfer by DFG.

in which the signal pulse is shaped.

The analysis presented in this section is similar to the frequency domain treatment used in Refs. 18 and 19. In order to simplify our analysis here, we consider a collinear geometry with a noncritical phase-matching condition, and assume plane-wave fields. We first introduce the notations to be used throughout this paper. The electric-field of an ultrashort pulse, in the time domain, is decomposed into its slowly and rapidly varying parts as follows:

$$
E_{j}(z, t)=A_{j}(z, t) \exp \left[i k\left(\omega_{j}\right) z-i \omega_{j} t\right]
$$

where $\omega_{j}$ is the carrier frequency of the pulse, with corresponding wavevector $k\left(\omega_{j}\right)$, and $A_{j}(z, t)$ is the slowly-varying complex envelope. The complex amplitude $A_{j}(z, t)$ can be written as:

$$
A_{j}(z, t)=\Psi_{j}(z, t) \exp \left[i \phi_{j}(z, t)\right]
$$

where $\Psi_{j}(z, t)$ is the temporal profile, while $\phi_{j}(z, t)$ is the temporal phase. The description of the electric field in the frequency domain is then given by its Fourier transform:

$$
\hat{E}_{j}\left(z, \Omega_{j}\right)=\mathcal{F}[E(z, t)]=\hat{A}_{j}\left(z, \Omega_{j}\right) \exp \left[i k\left(\omega_{j}+\Omega_{j}\right) z\right]
$$

where $\Omega_{j}=\omega-\omega_{j}$ is the frequency-detuning from the carrier frequency.

The simplest mathematical description of the generated idler pulse envelope from the DFG process in a dispersionless material, assuming undepleted pump and unamplified signal, is given by:

$$
A_{i}(L, t)=i \gamma_{i} A_{p}(0, t) A_{s}^{*}(0, t-\tau)
$$

where $\gamma_{i}=\frac{d \omega_{i}}{n_{i} c}$ is the nonlinear coupling coefficient, with $d$ being the effective nonlinear coefficient of the material, and $n_{i}$ its refractive index at $\omega_{i}$, while $\tau$ is a relative temporal delay between the input pump and signal pulses. In the frequency-domain, the above mathematical 
expression translates into the following convolution:

$$
\hat{A}_{i}\left(L, \Omega_{i}\right)=i \gamma_{i} \hat{A}_{p} \otimes \hat{A}_{s}^{*}=i \gamma_{i} \int_{-\infty}^{\infty} \hat{A}_{p}\left(0, \Omega_{s}+\Omega_{i}\right) \hat{A}_{s}^{*}\left(0, \Omega_{s}\right) \exp \left(-i \tau \Omega_{s}\right) d \Omega_{s} .
$$

The above convolution suggests that the parametric transfer itself is the fundamental limitation to the indirect pulse shaping. In general, the convolution limits the resolution at which the pump intensity and phase can be transferred onto the idler pulse. A perfect transfer $\left[\hat{A}_{i}\left(\Omega_{i}\right)=\hat{A}_{p}\left(\Omega_{i}\right)\right]$ can only happen when the signal is chosen to be a Dirac delta function $\left[\hat{A}_{s}\left(\Omega_{s}\right)=\delta\left(\Omega_{s}\right)\right]$, implying a continuous-wave, instead of a pulsed signal. In practice, however, signal pulses with a narrow spectrum relative to the shaped pump pulses are often sufficient. Additional requirements for the signal pulses can be drawn from the convolution. Firstly, the spectrum of the signal pulses is required to be symmetric in order to preserve the spectral intensity shape of the pump pulses. Secondly, the signal pulses have to be chirp-free to prevent unwanted chirp being transferred to the idler pulses, and finally, a full temporal overlap $(\tau=0)$ is required between the input pulses.

In this work, we are interested in the extent of the parametric transfer from the pump to the idler pulses. We define the following measure for the fidelity of parametric transfer in terms of intensity and phase:

$$
Z=1-\frac{\int S_{p}(\Omega, \tau ; z=0) S_{i}(\Omega, \tau ; z=L) d \Omega d \tau}{\left[\int S_{p}^{2}(\Omega, \tau ; z=0) d \Omega d \tau \int S_{i}^{2}(\Omega, \tau ; z=L) d \Omega d \tau\right]^{\frac{1}{2}}},
$$

where the polarization gate spectrogram $S(\Omega, \tau, z)$ is given by: ${ }^{20}$

$$
S_{j}(\Omega, \tau ; z)=\left.\left.\left|\int_{-\infty}^{\infty} A_{j}(z, t)\right| A_{j}(z, t-\tau)\right|^{2} \exp (i \Omega t) d t\right|^{2} .
$$

Transfer fidelity $Z$ essentially indicates the amount of overlap between the spectrograms of the output idler pulse and the input pump pulse. Its value ranges from 0 , indicating a perfect overlap, to 1 , indicating no overlap. It is worth noting that transfer fidelity $Z$ is independent of the numerical discretization size in the computation.

In general it is of more interest to transfer complex, non-transform-limited pulses and so, as an example, we will consider a Gaussian pump pulse that has undergone a self-phasemodulation in a Kerr-medium, in order to study the fidelity of parametric transfer via DFG. The pump pulse has the following form:

$$
A_{p}(t)=A_{p}^{(0)} f(t) \exp \left(i 10|f(t)|^{2}\right)
$$

where

$$
f(t)=\exp \left[-2 \ln 2\left(\frac{t}{\delta t_{p}}\right)^{2}\right]
$$




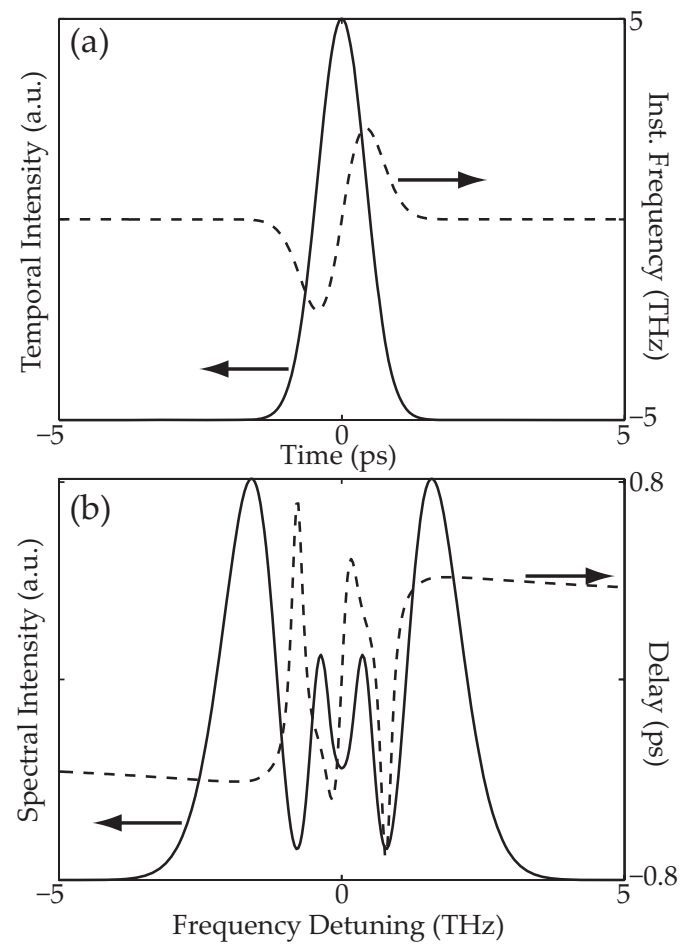

Fig. 2. a) Temporal intensity and instantaneous frequency, and b) spectral intensity and group delay of the input pump pulse. 

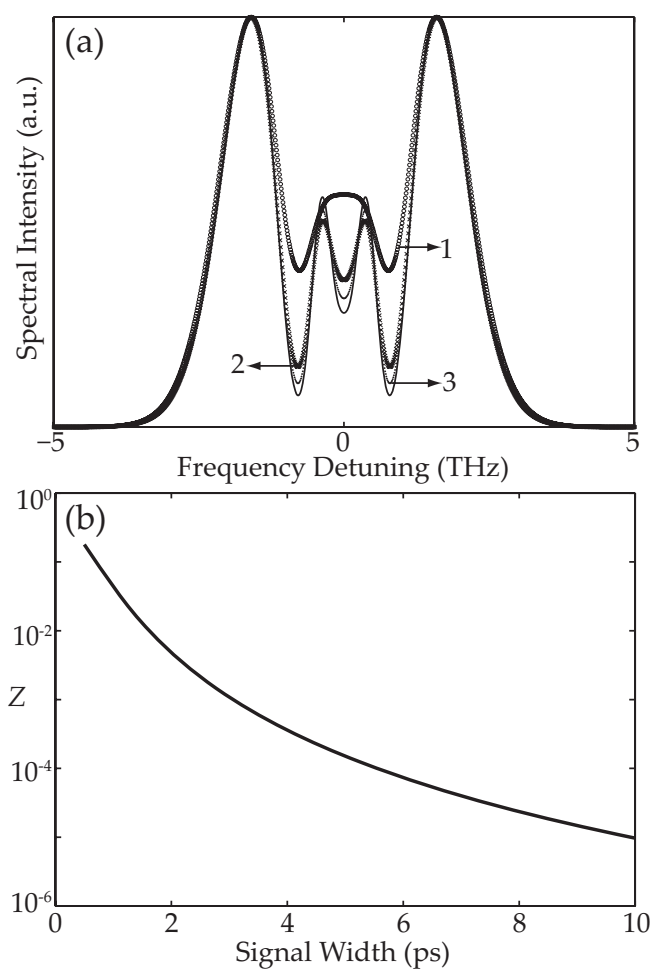

Fig. 3. a) Spectral intensities of the generated idler pulses from the interaction between pump pulses as described in the text and transform-limited Gaussian signal pulses of temporal FWHM shown by the numbers in picoseconds. b) Transfer fidelity $Z$ in logarithmic scale as a function of the signal pulsewidth.

$A_{p}^{(0)}$ denotes the pump envelope peak, and $\delta t_{p}$ is the temporal full-width half-maximum (FWHM). Figure 2 shows the temporal intensity, instantaneous frequency, spectral intensity, and the spectral group delay of the pump pulse. Note that the leading side of the pump pulse has a positive instantaneous frequency, corresponding to the spectral part in the positive frequency detuning, while the trailing side has a negative instantaneous frequency, corresponding to the spectral part in the negative frequency detuning. We chose a temporal FWHM of $1 \mathrm{ps}$, leading to a spectral FWHM of $4.44 \mathrm{THz}$, equivalent to $\sim 16 \mathrm{~nm}$ for a central wavelength at around $1 \mu \mathrm{m}$. Such a spectral FWHM is able to support transform-limited Gaussian pulses with a temporal FWHM of 100 fs.

When we calculated the generated idler pulse through the convolution (Eq. 5) of the above input pump pulse and transform-limited Gaussian signal pulses of different temporal FWHM, we obtain Fig. 3. As one can easily observe, longer duration signal pulses (narrower bandwidths) yield a lower $Z$, indicating a better fidelity transfer. Figure 3(a) shows the generated 
idler spectrum using signal pulses with temporal (spectral) FWHM of $1 \mathrm{ps}(0.44 \mathrm{THz}), 2 \mathrm{ps}$ $(0.22 \mathrm{THz})$, and $3 \mathrm{ps}(0.15 \mathrm{THz})$, leading to a transfer fidelity $Z$ of $4.38 \times 10^{-2}, 4.83 \times 10^{-3}$, and $1.08 \times 10^{-3}$, respectively. Since the parametric transfer cannot be perfectly achieved, we have to establish a limit to $Z$ that we deem as an acceptable level of transfer fidelity. We therefore chose the value $Z<5 \times 10^{-3}$ as a condition for a good parametric transfer. In practice, the choice of the signal spectral FWHM depends on the finest spectral structure of the shaped pump pulse that we wish to transfer.

\section{Theoretical Analysis of Parametric Transfer in Dispersive Media}

In the previous section, we have established the fundamental limitations and requirements for a good fidelity parametric transfer through DFG. However, the simple convolution, on which the analysis was based, only arises from interactions in a non-dispersive nonlinear material, i.e. a very short length of material, and by using relatively low input powers in order to avoid pump depletion and signal amplification. To implement a situation in which such assumptions were valid would limit the conversion efficiency of the generated idler pulses, and thus the amount of power that can be put into practical uses.

When a longer second-order nonlinear material is used to increase the conversion efficiency, chromatic dispersion starts to affect the interaction in a complicated manner, especially since this technique involves interactions between pulses in different spectral regions. Chromatic dispersion causes the envelope of each of the interacting pulses to propagate at different group

velocity $u=\left[\frac{d k(\omega)}{d \omega}\right]^{-1}$, leading to a temporal walk-off. Chromatic dispersion further causes different spectral components within a pulse to travel at different speed, the phenomenon called of group velocity dispersion (GVD), whose parameter is given by $\beta=\frac{d^{2} k(\omega)}{d \omega^{2}}$. As we shall see later, temporal walk-off effects and GVD play an important role in the parametric transfer, and so they are included in the following analysis.

The generated idler spectral envelope, after propagating in a nonlinear crystal of length $L$, and under the assumption of negligible pump-depletion and signal-amplification, is given by: $:^{18,19,21}$

$$
\frac{\partial \hat{A}_{i}\left(z, \Omega_{i}\right)}{\partial z}=i \gamma_{i} \int_{-\infty}^{\infty} \hat{A}_{p}\left(\Omega_{s}+\Omega_{i}\right) \hat{A}_{s}^{*}\left(\Omega_{s}\right) \exp \left(i \tau \Omega_{s}\right) \exp \left[i \Delta k\left(\Omega_{s}, \Omega_{i}\right) z\right] d \Omega_{s},
$$

where

$$
\Delta k\left(\Omega_{s}, \Omega_{i}\right)=k\left(\omega_{p}+\Omega_{s}+\Omega_{i}\right)-k\left(\omega_{s}+\Omega_{s}\right)-k\left(\omega_{i}+\Omega_{i}\right)
$$

is the wavevector mismatch between the interacting pulses. This wavevector can be expanded in a Taylor series to yield:

$$
\Delta k\left(\Omega_{s}, \Omega_{i}\right)=\Delta k_{0}+\delta \nu_{p s} \Omega_{s}+\delta \nu_{p i} \Omega_{i}+\frac{1}{2} \delta \beta_{p s} \Omega_{s}^{2}+\frac{1}{2} \delta \beta_{p i} \Omega_{i}^{2}+\beta_{p} \Omega_{s} \Omega_{i}+\mathcal{O}\left(\Omega_{s}^{3}, \Omega_{i}^{3}\right),
$$


where $\Delta k_{0}=k\left(\omega_{p}\right)-k\left(\omega_{s}\right)-k\left(\omega_{i}\right)$ is the wavevector mismatch between the carrier frequencies of the interacting pulses, $\delta \nu_{p s}=\frac{1}{u_{p}}-\frac{1}{u_{s}}$ is the group velocity mismatch (GVM) between pump and signal pulses, $\delta \nu_{p i}=\frac{1}{u_{p}}-\frac{1}{u_{i}}$ is the GVM between pump and idler pulses, $\beta_{p}$ is the GVD of the pump pulse, $\delta \beta_{p i}=\beta_{p}-\beta_{i}$ is the GVD mismatch between pump and idler pulses, and $\delta \beta_{p s}=\beta_{p}-\beta_{s}$ is the GVD mismatch between pump and signal pulses. We are interested in phase-matched interactions, i.e. $\Delta k_{0}=0$, so we shall ignore the wavevector mismatch in the remainder of this section.

Substituting the above Taylor expansion into Eq. 10, and ignoring GVD and higher-order terms, we arrive at the following expression:

$$
\frac{\partial \hat{A}_{i}\left(z, \Omega_{i}\right)}{\partial z}=i \gamma_{i} \int_{-\infty}^{\infty} \hat{A}_{p}\left(\Omega_{s}+\Omega_{i}\right) \hat{A}_{s}^{*}\left(\Omega_{s}\right) \exp \left(i \tau \Omega_{s}\right) \exp \left[i\left(\delta \nu_{p s} \Omega_{s}+\delta \nu_{p i} \Omega_{i}\right) z\right] d \Omega_{s} .
$$

The analytical integration of the above equation will result in a complicated expression, mainly because of the exponential term containing the GVM parameters. We shall consider the effect of each parameter separately, treating them as two special cases. First, when the GVM between pump and signal pulses $\delta \nu_{p s}$ can be ignored, Eq. 13 becomes:

$$
\frac{\partial \hat{A}_{i}\left(z, \Omega_{i}\right)}{\partial z}=i \gamma_{i} \int_{-\infty}^{\infty} \hat{A}_{p}\left(\Omega_{s}+\Omega_{i}\right) \hat{A}_{s}^{*}\left(\Omega_{s}\right) \exp \left(i \tau \Omega_{s}\right) \exp \left[i \delta \nu_{p i} \Omega_{i} z\right] d \Omega_{s}
$$

from which one can easily see that exponential term does not depend on $\Omega_{s}$, and thus can be moved out of the integration. Taking the integration of both sides with respect to $z$ results in:

$$
\hat{A}_{i}\left(L, \Omega_{i}\right)=D\left(L, \Omega_{i}\right)\left[\hat{A}_{p} \otimes \hat{A}_{s}^{*}\right]
$$

where

$$
\begin{aligned}
D\left(L, \Omega_{i}\right) & =i \gamma_{i} \int_{0}^{L} \exp \left[i \delta \nu_{p i} \Omega_{i} z\right] d z \\
& =i \gamma_{i} \operatorname{sinc}\left[\delta \nu_{p i} \Omega_{i} \frac{L}{2}\right] \exp \left[i \delta \nu_{p i} \Omega_{i} \frac{L}{2}\right]
\end{aligned}
$$

Equation 15 describes a filter function $\left[D\left(L, \Omega_{i}\right)\right]$ acting on a convolution. The filter function has a sinc shape, whose FWHM is given by $\delta f=0.88 /\left(\delta \nu_{p i} L\right)$. When the filter bandwidth is much larger than the FWHM of the pulse spectrum, it hardly affects the parametric transfer. However, when the filter bandwidth is comparable to the pulse spectrum, spectral clipping will occur, thus degrading the parametric transfer. Therefore, it is imperative to work with a condition at which the bandwidth of the filter function is much larger than the spectral FWHM of the shaped pump pulse in order to achieve good fidelity spectral transfer.

The second case is negligible $\delta \nu_{p i}$, such that Eq. 13 becomes:

$$
\frac{\partial \hat{A}_{i}\left(z, \Omega_{i}\right)}{\partial z}=i \gamma_{i} \int_{-\infty}^{\infty} \hat{A}_{p}\left(\Omega_{s}+\Omega_{i}\right) \hat{A}_{s}^{*}\left(\Omega_{s}\right) \exp \left(i \tau \Omega_{s}\right) \exp \left[i \delta \nu_{p s} \Omega_{s} z\right] d \Omega_{s} .
$$


In contrast to the previous case, the integration of the exponential term with respect to $z$ must be carried out within the $\Omega_{s}$ integration, resulting in the following convolution:

$$
\hat{A}_{i}\left(L, \Omega_{i}\right)=\hat{A}_{p} \otimes \hat{A}_{e}
$$

where

$$
\begin{aligned}
\hat{A}_{e}\left(\Omega_{s}\right) & =i \gamma_{i} \hat{A}_{s}^{*}\left(\Omega_{s}\right) \exp \left(i \tau \Omega_{s}\right) \int_{0}^{L} \exp \left[i \delta \nu_{p s} \Omega_{s} z\right] d z \\
& =i \gamma_{i} \operatorname{sinc}\left[\delta \nu_{p s} \Omega_{s} \frac{L}{2}\right] \hat{A}_{s}^{*}\left(\Omega_{s}\right) \exp \left[i\left(\tau+\frac{\delta \nu_{p s} L}{2}\right) \Omega_{s}\right] .
\end{aligned}
$$

The noteworthy contrast between Eqs. 15 and 18 is the lack of the filter function in the latter. The latter is only a convolution between the pump pulse and the so-called effective signal pulse $\hat{A}_{e}\left(\Omega_{s}\right)$, which is essentially a time-delayed filtered signal pulse. It then follows directly that good fidelity parametric transfer can be achieved if the effective signal pulse meets the requirements mentioned in the previous section. Since sinc is an even function, the effective signal pulse is guaranteed to be symmetric provided the signal pulse is an even function as well. Furthermore, as the effective signal pulse has to be fully temporally overlapped with the pump pulse, a time delay of $\tau=\tau_{c}=-\delta \nu_{p s} L / 2$ has to be introduced between the input pump and signal pulses. The sinc filter function in this case actually improves the fidelity of the parametric transfer because as it becomes narrower, it reduces the spectral FWHM of the original signal pulse.

The analysis in this section suggests that the conditions for a good fidelity parametric transfer in a dispersive material is to choose a working condition for which the $\delta \nu_{p i} L$ does not cause significant spectral clipping, and to introduce a temporal delay between the input pump and signal pulses to offset their temporal walk-off.

\section{Numerical Analysis of Parametric Transfer}

The frequency-domain analysis in the previous section provides us with a rather qualitative understanding of parametric transfer via DFG. One can obtain a better picture by performing an extensive numerical simulation based on the following set of coupled equations for the DFG interaction in time domain:

$$
\begin{aligned}
& \frac{\partial A_{p}}{\partial z}+\frac{1}{u_{p}} \frac{\partial A_{p}}{\partial t}+\frac{i \beta_{p}}{2} \frac{\partial^{2} A_{p}}{\partial t^{2}}=i \gamma_{p} A_{s} A_{i} \exp \left(-i \Delta k_{0} z\right), \\
& \frac{\partial A_{s}}{\partial z}+\frac{1}{u_{s}} \frac{\partial A_{s}}{\partial t}+\frac{i \beta_{s}}{2} \frac{\partial^{2} A_{s}}{\partial t^{2}}=i \gamma_{s} A_{p} A_{i}^{*} \exp \left(i \Delta k_{0} z\right), \\
& \frac{\partial A_{i}}{\partial z}+\frac{1}{u_{i}} \frac{\partial A_{i}}{\partial t}+\frac{i \beta_{i}}{2} \frac{\partial^{2} A_{i}}{\partial t^{2}}=i \gamma_{i} A_{p} A_{s}^{*} \exp \left(i \Delta k_{0} z\right),
\end{aligned}
$$

where $\gamma_{j}=\frac{d \omega_{j}}{n_{j} c}$ is the nonlinear coupling coefficient, $u$ is the group velocity, $\beta$ is the GVD parameter, and the subscript $j=p, s, i$ denotes the pump, signal and idler pulses, respectively. 


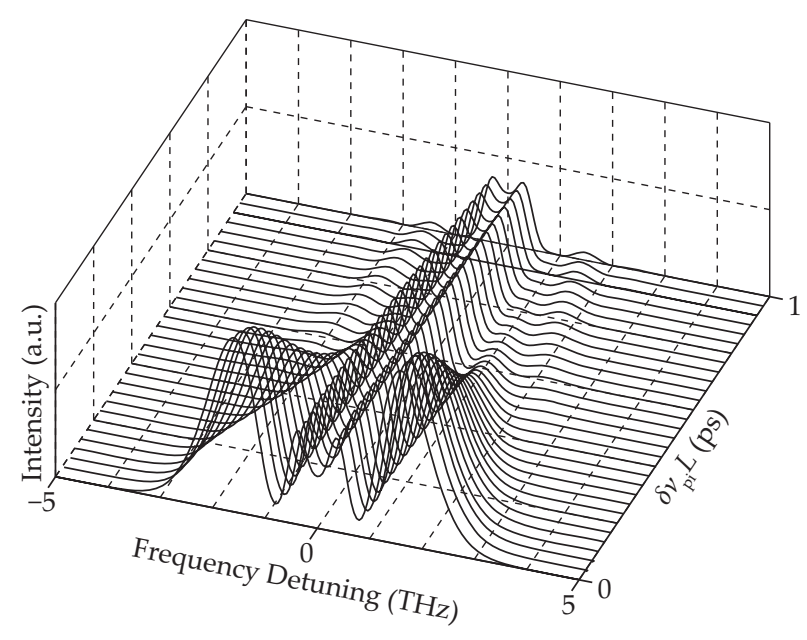

Fig. 4. Generated idler spectra as a function of $\delta \nu_{p i} L$ at $\delta \nu_{p s}=0$ and $\tau=0$.

It is to be understood that in the above set of equations, $A_{p}=A_{p}(z, t), A_{s}=A_{s}(z, t-\tau)$, and $A_{i}=A_{i}(z, t)$. Equation 20 includes material dispersion up to GVD, nonlinear evolution of the pump and signal pulses, and the phase-mismatch term. We solved the above set of equations using the symmetric split-step Fourier method ${ }^{22}$ with a Runge-Kutta integrator, in the pump pulse's frame of reference. We shall assume, in this section, that the phase-matching condition is achieved, i.e. $\Delta k_{0}=0$, through appropriate phase-matching methods. The nonlinear coupling coefficients used in our numerical simulations were $\gamma_{p}=4.42 \times 10^{-5} \mathrm{~V}^{-1}$, $\gamma_{s}=3.11 \times 10^{-5} \mathrm{~V}^{-1}, \gamma_{i}=1.38 \times 10^{-5} \mathrm{~V}^{-1}$, which corresponds to bulk periodically-poled lithium niobate (PPLN). The length of the material was chosen to be $1 \mathrm{~cm}$, unless stated otherwise. The signal pulse used was a transform-limited Gaussian with a temporal FWHM of $4 \mathrm{ps}$, yielding a parametric transfer fidelity $Z=3.6 \times 10^{-4}$ for interactions in a dispersionless material, without pump-depletion and signal amplification.

\section{A. Effect of Temporal Walk-Off}

Initially, we verify the frequency-domain analysis presented in the previous section by performing numerical simulations using low input pump and signal intensities, and omitting GVD and higher order dispersion in these calculations. Here, we shall first consider the case of $\delta \nu_{p s}=0$. Figure 4 shows the generated idler spectra calculated from our numerical simulations as a function of $\delta \nu_{p i} L$ while both input pulses are fully temporally overlapped $(\tau=0)$. As $\delta \nu_{p i} L$ increases, the width of the filter decreases, resulting in spectral clipping. While the middle part of the spectrum remains constant, the spectral intensity of the two main side lobes decreases, and additional side lobes start to appear in exchange, showing an evidence 


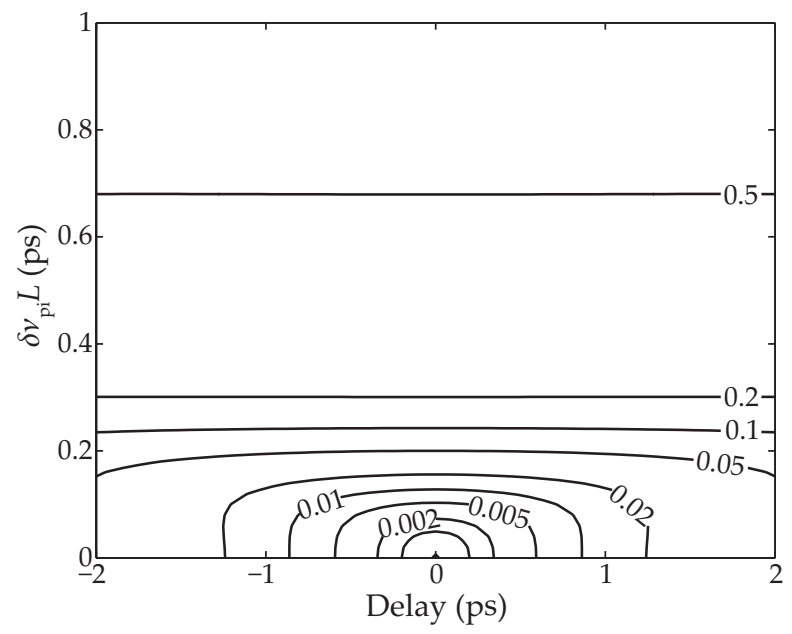

Fig. 5. Contour plot of transfer fidelity $Z$ as a function of $\delta \nu_{p i} L$ and $\tau$ with $\delta \nu_{p s}=0$.

of the sinc shape of the filter function in Eq. 15. Figure 5 shows the contour plot of the calculated transfer fidelity $Z$ as a function of $\delta \nu_{p i} L$ and $\tau$. As expected from our analysis in the previous section, the transfer fidelity rapidly degrades as $\delta \nu_{p i} L$ increases. The upper bound of $\delta \nu_{p i} L$ that still gives an acceptable fidelity of parametric transfer corresponds to the filter FWHM of 1.5 times that of the pump spectral FWHM, corresponding to a total walk-off of $132 \mathrm{fs}$ between the input pump and idler pulses.

We now consider the case of $\delta \nu_{p i}=0$. Figure 6 shows the generated idler spectra calculated from our numerical simulations for $\delta \nu_{p s} L=0,0.5,1 \mathrm{ps}$, and the input pulses are fully temporally overlapped $(\tau=0)$. One can easily see that the general shape of the idler spectrum remains constant as $\delta \nu_{p s} L$ varies. The interesting point in this case is that as $\delta \nu_{p s} L$ increases, the spectral intensity in the negative frequency detuning decreases, while the intensity in the positive frequency detuning increases, breaking the symmetry of the generated idler spectrum. This can be explained by looking at the propagation in the time domain. Since $\delta \nu_{p s} L>0$, signal pulses travel with a higher group velocity than the pump pulses, and they will walk through the pump pulses during the propagation in the material, implying that the temporal overlap is shifted toward the leading side of the pump pulses. The leading side of the pump pulses, as we recall from Fig. 2, has a positive instantaneous frequency, which corresponds to the spectral part in the positive frequency detuning. Since the temporal overlap is shifted toward the leading side of the pump pulses, the spectral part in the positive frequency detuning is converted toward the idler pulses more efficiently than the part in the negative frequency detuning, as confirmed by Fig. 6 . Figure 7 shows the contour plot of the 


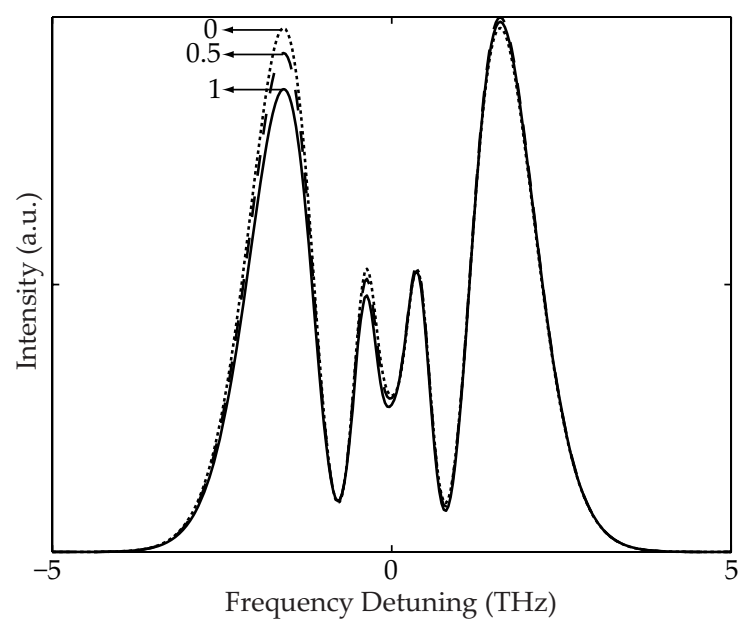

Fig. 6. Generated idler spectra at $\delta \nu_{p i}=0$ and $\tau=0$ for $\delta \nu_{p s} L=0,0.5,1 \mathrm{ps,}$ as indicated by the numbers.

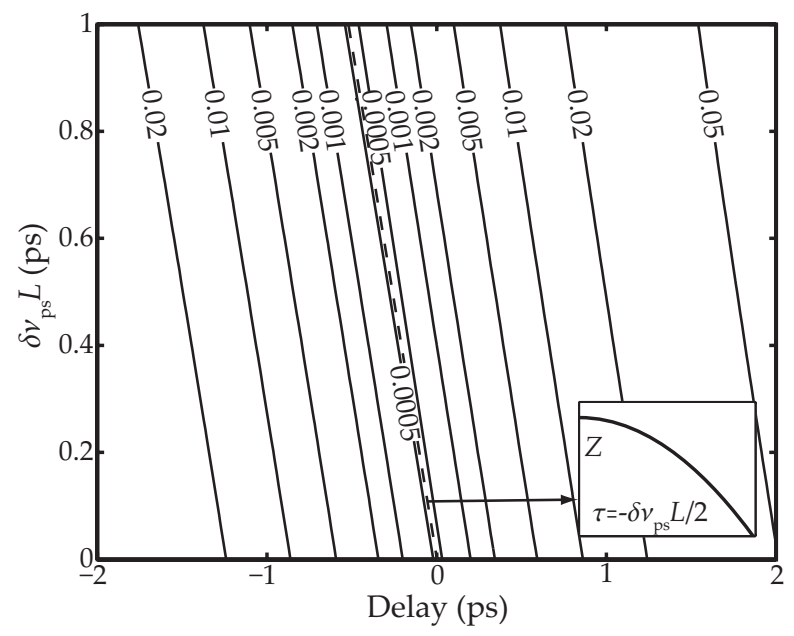

Fig. 7. Contour plot of transfer fidelity $Z$ as a function of $\delta \nu_{p s} L$ and $\tau$ with $\delta \nu_{p i}=0$. The inset shows $Z$ along $\tau=-\delta \nu_{p s} L / 2$ as indicated by the dashed line. Note that this line is not part of the contour plot, i.e. it does not show a constant value of $Z$. 


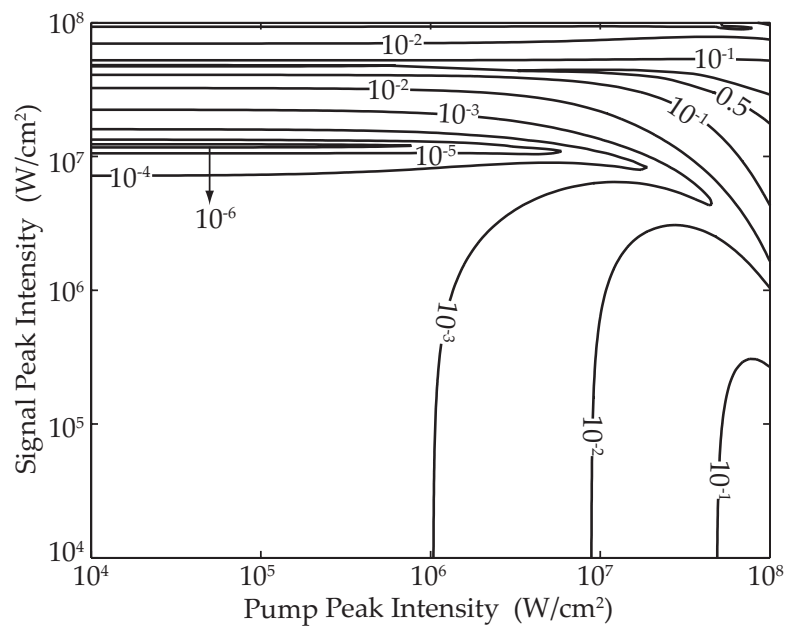

Fig. 8. Contour plot of transfer fidelity $Z$ as a function of input pump and signal peak powers with $\delta \nu_{p s}=\delta \nu_{p i}=0$, and $\tau=0$.

calculated transfer fidelity $Z$ as a function of $\delta \nu_{p s} L$ and $\tau$. In contrast to the previous case, there exist points in the $\tau$ and $\delta \nu_{p s} L$ space that minimizes $Z$. These points form a line which corresponds to a time delay of $\tau_{c}$ between the input pulses. Furthermore, the transfer fidelity along this line improves as $\delta \nu_{p s} L$ increases, as shown by the inset of Fig. 7 . These results are in excellent agreement with the analysis presented in the previous section.

\section{B. Effect of Pump Depletion and Signal Amplification}

In addition to increasing the crystal length in order to produce more photons at the idler wavelength, one can also increase the input intensities. However, in doing so, the pump pulse may experience significant depletion, while the signal pulse may experience significant amplification. These conditions result in the generated idler pulses not being proportional to the convolution of the input pulses, and thus degrades the parametric transfer. We shall analyze the effect of the intensity variation without material dispersion, so that the temporal derivatives in Eq. 20 can be ignored. Figure 8 shows the contour plot of the transfer fidelity $Z$ as a function of both input pump and signal peak intensities. This figure shows that the peak intensities of input pump pulses up to $\sim 8 \mathrm{MW} / \mathrm{cm}^{2}$ and input signal pulses up to $\sim 10 \mathrm{MW} / \mathrm{cm}^{2}$ yield an acceptable parametric transfer. Figure 9 (a) shows the transfer fidelity $Z$ as a function of signal peak intensity $I_{s}^{(0)}$ with input pump peak intensity being kept constant at $I_{p}^{(0)}=10 \mathrm{~kW} / \mathrm{cm}^{2}$. In this condition, there is negligible signal amplification for all values of signal peak intensities. The figure also shows the amount of depletion, $1-\frac{U_{p}(L)}{U_{p}(0)}$, experienced by the pump pulse, where $U(z)=\int I(z, t) d t$ is the pulse energy. As the input 

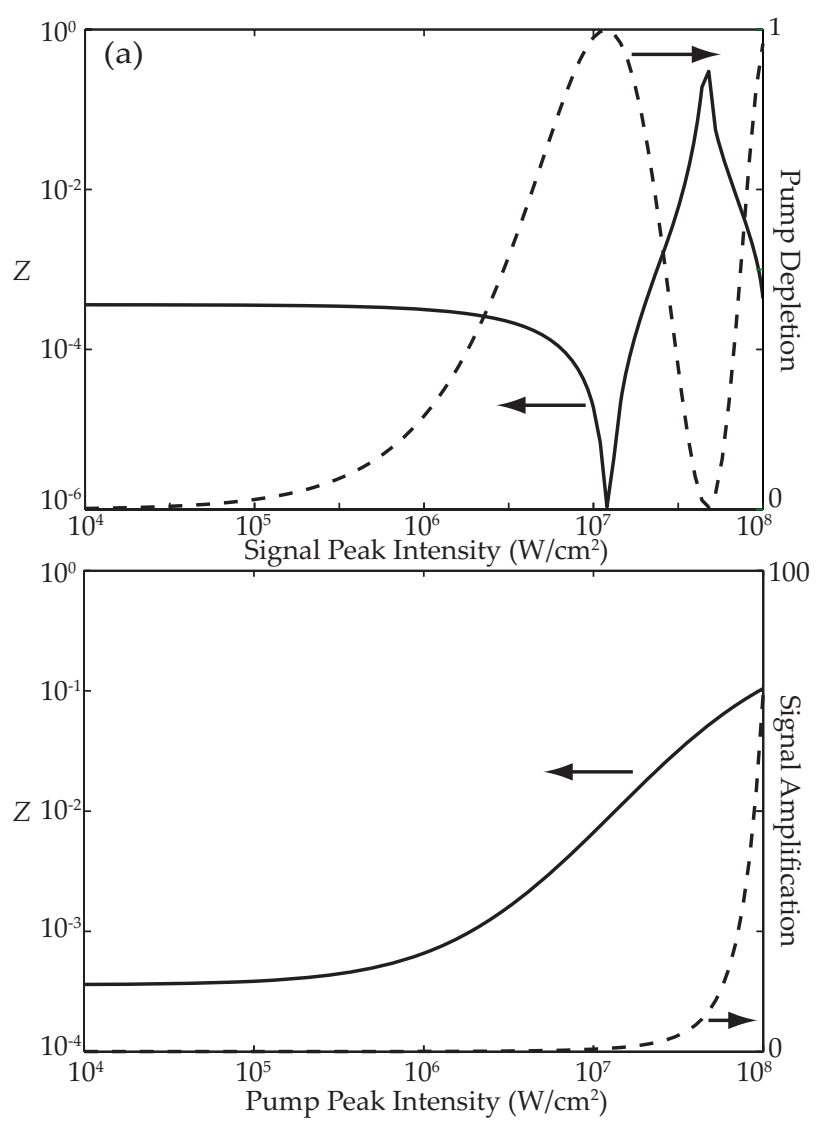

Fig. 9. a) Transfer fidelity $Z$ and pump depletion as a function of signal peak intensity, with input pump peak intensity being kept constant $10 \mathrm{~kW} / \mathrm{cm}^{2}$. b) Transfer fidelity $Z$ and signal amplification as a function of pump peak intensity, with input signal peak intensity being kept constant $10 \mathrm{~kW} / \mathrm{cm}^{2}$. 
signal peak intensity increases, the pump depletes, and $Z$ tends to be constant for several orders of magnitude, and then decreases, indicating an improvement in the transfer fidelity. This continues until the pump pulse almost completely depletes, which corresponds to the minimum value of transfer fidelity $Z$ in the picture. After the depletion, back-conversion happens, and $Z$ starts to increase again, indicating a degradation of the transfer fidelity. As the pump intensity used in the simulation of Fig. 9(a) was such that the signal amplification was negligible, we can ignore the equation for signal pulse evolution in Eq. 20, and analytically solve the remaining two equations to yield the following $z$ dependence of the idler pulse envelope:

$$
A_{i}(z, t)=i A_{p}(0, t) \sqrt{\frac{\gamma_{i}}{\gamma_{p}}} \sin \left[\sqrt{\gamma_{i} \gamma_{p}}\left|A_{s}(0, t)\right| z\right] \exp \left[-i \phi_{s}(0, t)\right]
$$

When one examines the above solution, the fact that there is a region of input signal peak intensities where the transfer fidelity $Z$ remains almost constant is obvious, since $A_{i}(L, t) \propto A_{p}(0, t)$, and $A_{s}(0, t)$ has been chosen to be wider than $A_{p}(0, t)$. The improvement of parametric transfer fidelity, indicated by the decrease of transfer fidelity $Z$, when the pump pulse further depletes, can be explained by the fact that the signal envelope $\left|A_{s}(0, t)\right|$ is inside a sine function in the above equation. As the signal pulse peak intensity is increased, the peak is the part of the pulse that will cause the argument of the sine function to approach $\pi / 2$ first. Since the gradient of the sine function around $\pi / 2$ is low, the change of the sine term around the high intensity part will be low. Therefore, this term results in an artificial broadening of the signal pulse as its peak intensity is increased, and thus justifies the improvement of the parametric transfer fidelity. When the peak signal intensity makes the argument of the sine function equal to $\pi / 2$, the pump almost completely depletes, especially as it is narrower than the signal pulse. This condition corresponds to the best transfer fidelity in Fig. 9(a).

Figure 9 (b) shows the transfer fidelity $Z$ as a function of pump peak intensity with input signal peak intensity being kept constant at $10 \mathrm{~kW} / \mathrm{cm}^{2}$. In this condition, there is negligible pump depletion for all value of signal pump intensities. The figure also shows the amount of amplification, $\frac{U_{s}(L)}{U_{s}(0)}-1$, experienced by the signal pulse. As the input signal peak intensity increases, the signal was amplified, and correspondingly, transfer fidelity $Z$ increases monotonically. As the signal intensity used in the simulation of Fig. 9(b) was such that the pump depletion was negligible, we can ignore the equation for pump pulse evolution in Eq. 20, and solve analytically the remaining two equations to yield the following $z$ dependence of the idler pulse envelope:

$$
A_{i}(z, t)=i \sinh \left[\sqrt{\gamma_{i} \gamma_{s}}\left|A_{p}(0, t)\right| z\right] \exp \left[i \phi_{p}(0, t)\right] \sqrt{\frac{\gamma_{i}}{\gamma_{s}}} A_{s}^{*}(0, t) .
$$

In contrast to the previous case, $A_{i}(L, t)$ is not directly proportional to $A_{p}(0, t)$. Instead, the pump pulse envelope becomes the argument of a hyperbolic sine function. For a small gain, 
$\sinh (x) \approx x$, explaining the fact that $Z$ does not change over several orders of magnitude of pump peak intensity variations, where there is only a small gain. However, as the pump peak intensity keeps increasing, signal amplification becomes larger, and thus the parametric transfer fidelity degrades quickly.

So far, we have seen the effect of temporal walk-off, pump depletion and signal amplification on the fidelity of the parametric transfer. These effects, however, have been investigated separately from each other. The interaction of the pulses becomes more complicated when both the nonlinear intensity evolutions and temporal walk-off effects are all present. We now investigate the ability of the relative delay introduction between the input pulses to compensate GVM between pump and signal pulses in the presence of pump depletion or signal amplification. We performed numerical simulations with $\delta \nu_{p i}=0$ and $\delta \nu_{p s} L=1$ ps. Figure 10(a) shows the transfer fidelity $Z$ as a function of input signal peak intensities $I_{s}^{(0)}$ and temporal delay $\tau$. The input pump peak intensity was kept constant at $I_{p}^{(0)}=10 \mathrm{~kW} / \mathrm{cm}^{2}$. This choice of parameters results in a pump depletion, similar to Fig. 9(a), which does not significantly affect the parametric transfer. Hence, the fact that the required delay to offset the GVM between pump and signal pulses does not deviate from $\tau_{c}$ can be expected, which is the case shown in Fig. 10(a). Figure 10(b) shows the contour plot of transfer fidelity $Z$ as a function of input pump peak intensities $I_{p}^{(0)}$ and temporal delay $\tau$. The input signal intensity was kept constant at $I_{s}^{(0)}=10 \mathrm{~kW} / \mathrm{cm}^{2}$. The temporal delay between input pulses that minimizes transfer fidelity $Z$ is also shown in the figure. In the low input pump peak intensity regime, where there is negligible signal amplification, the delay which minimizes $Z$ is given by $\tau_{c}$, as we have seen before. As the input pump peak intensity is increased, signal amplification takes place, degrading the parametric transfer. A larger delay is then required to maintain high parametric transfer fidelity, implying that the interaction happens with the lower intensity part of the signal pulse, i.e. the leading part of the pulse, in order to minimize signal amplification.

\section{C. Effect of Group Velocity Dispersion}

We now discuss the effect of GVD on the parametric transfer fidelity. Reexamining Eqs. 10 and 12 , the effect of GVD is governed by three parameters: $\beta_{p}, \delta \beta_{p s}$, and $\delta \beta_{p i}$. Of these three parameters, we can neglect $\delta \beta_{p s}$, because we have chosen the signal pulse to be sufficiently wide, and both the input pump and signal pulses are in the NIR region, leaving us with only two parameters to be investigated. Since $\delta \beta_{p i}$ is related to the $\Omega_{i}^{2}$ factor (see Eq. 12), it can be moved outside the integration in Eq. 10. The integration with respect to $z$ will yield a filter function with a linear group delay, a similar expression to Eq. 16. This linear group delay can be compensated by introducing an opposite group delay onto the input pump or the output idler pulses in order to to compensate the effect of GVD, i.e. pre- or post-compensation. 

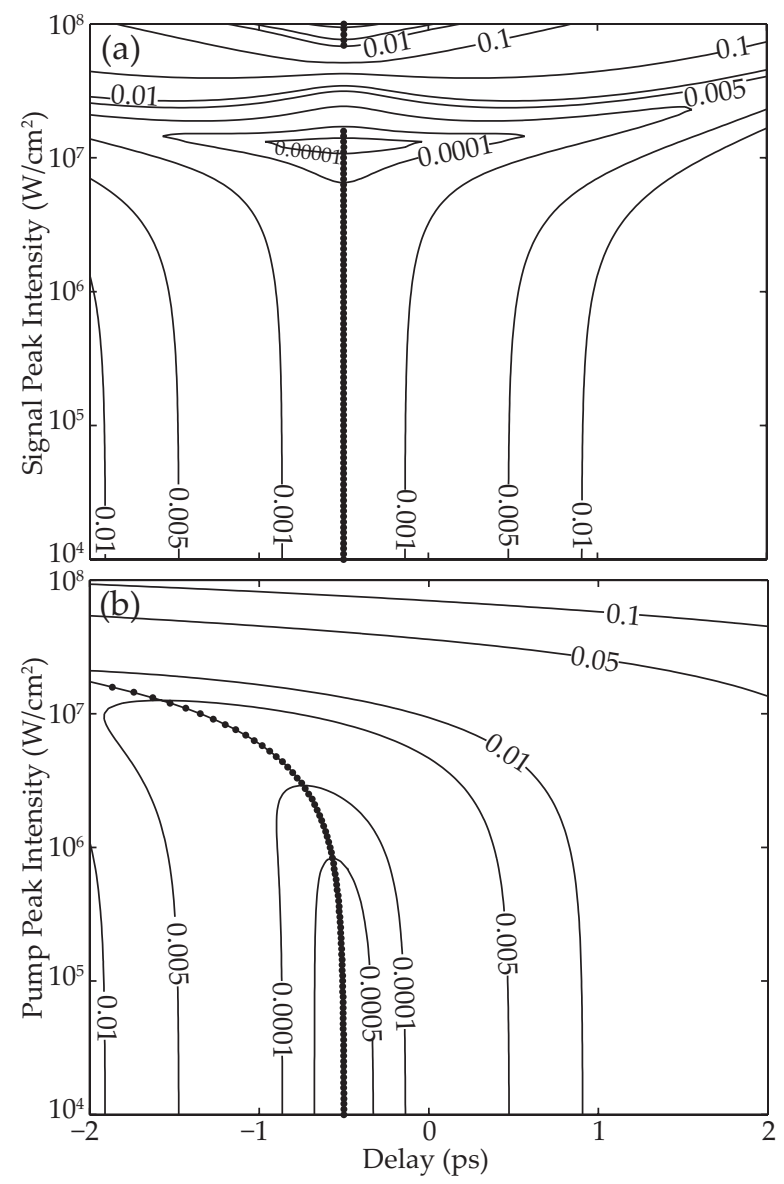

Fig. 10. Contour plot of transfer fidelity $Z$ as a function of relative delay $\tau$ between input pulses, and peak intensities of (a) pump pulses, and (b) signal pulses. Temporal delay which minimizes $Z$ for every power level are shown by the dots. 


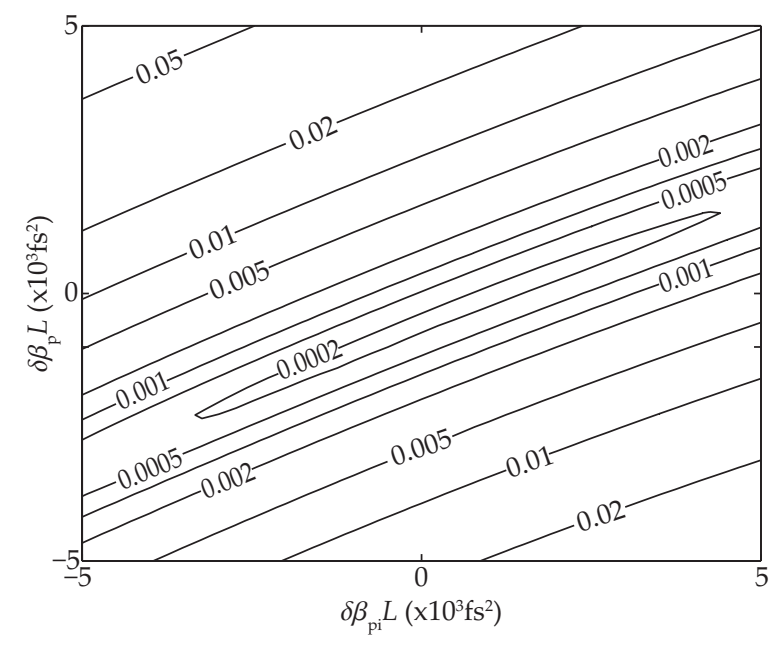

Fig. 11. Contour plot of transfer fidelity $Z$ as a function of $\beta_{p} L$ and $\delta \beta_{p i} L$.

Either scheme or the combination of both yield the same result. In practice, the required compensation can be included directly in the shaped input pulse, but in the strict context of parametric transfer, we would no longer be looking at getting the best replication of the shaped pump pulse but rather the best production of a certain target pulse. Meanwhile, since $\beta_{p}$ is related to the mixed $\Omega_{s} \Omega_{i}$ term, Eq. 10 would not reduce to a simple convolution, and thus would significantly degrade the parametric transfer. Due to the mixed term, it is also expected that the required delay $\tau$ to compensate $\delta \nu_{p s}$ is not equal to $\tau_{c}$.

Figure 11 shows contour plot of the calculated transfer fidelity $Z$ as a function of $\beta_{p} L$ and $\delta \beta_{p i} L$, with $\delta \nu_{p i}=0, \delta \nu_{p s} L=1 \mathrm{ps}$, and $I_{p}^{(0)}=I_{p}^{(0)}=10 \mathrm{~kW} / \mathrm{cm}^{2}$. In calculating $Z$, it was necessary to implement an optimization algorithm to locate the delay $\tau$ which compensates $\delta \nu_{p s}$ and some of the GVD. The algorithm we chose was the Nelder-Mead algorithm. ${ }^{23}$ From the figure, it can be easily seen that the gradient of $Z$ along $\beta_{p} L$ is higher than that along $\delta \beta_{p i} L$. Furthermore, it can be observed that there exists a line of minimum $Z$. This line, however, does not coincide with the origin, i.e. $\left(\beta_{p} L, \delta \beta_{p i} L\right)=(0,0)$. Because the resolution of the transferred phase is limited by the convolution, this result implies that the introduction of group delay in the material by GVD may improve the transfer fidelity. It is worth noting that the result would depend on the group delay of the pump pulse one wishes to transfer. A similar picture to Fig. 11 can be obtained for higher input pump and signal peak intensities. 


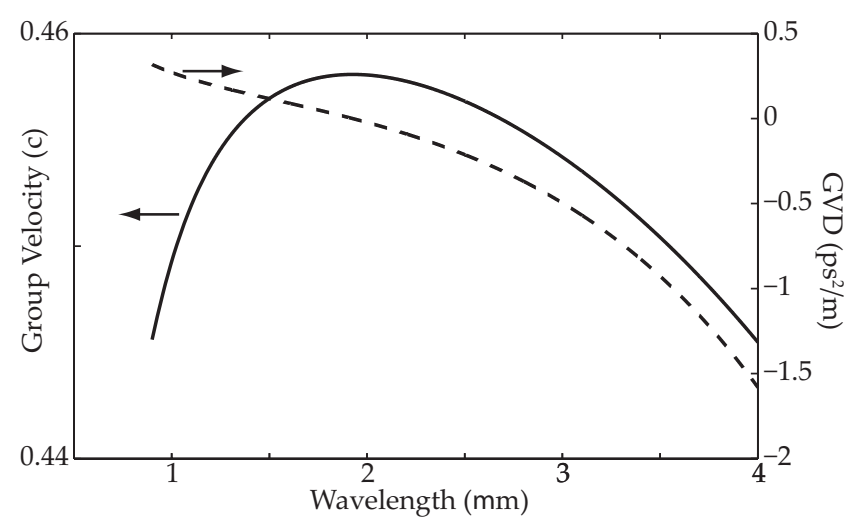

Fig. 12. Group velocity (solid curve) and group velocity dispersion (GVD, dashed curve) of lithium niobate at a temperature of $T=120{ }^{\circ} \mathrm{C}$ as calculated from the Sellmeier equation. ${ }^{24}$

\section{Parametric Transfer via DFG in PPLN}

We have investigated the effects of several parameters on the parametric transfer fidelity in the previous sections. In this section, we shall numerically investigate the parametric transfer fidelity in a real material. In order to have the condition of no temporal walk-off between the pump and idler pulses, the GVM between the pump and idler pulses must be zero $\left(\delta \nu_{p i}=0\right)$, implying the group velocity profile has to have a turning point, i.e. a zero GVD, at a wavelength between the pump and idler wavelengths. Chromatic dispersion of lithium niobate, shown in Fig. 12, suggests that there exist combinations of pump wavelengths around $1 \mu \mathrm{m}$ and idler carrier wavelengths around $3.5 \mu \mathrm{m}$ which satisfy $\delta \nu_{p i}=0$. Correspondingly, the signal wavelength is around $1.5 \mu \mathrm{m}$. Quasi-phase-matching techniques can be used to phase-match these wavelengths. The ready availability of laser sources with a wavelength around $1 \mu \mathrm{m}$ and $1.5 \mu \mathrm{m}$ makes the choice of periodically-poled lithium niobate (PPLN) natural for producing shaped MIR pulses in the $3-4 \mu \mathrm{m}$ regime. Furthermore, this material has been widely used for frequency conversion and optical parametric oscillator devices, and its fabrication techniques have been well established. We numerically solved Eq. 20 using dispersion parameters (GVM and GVD) of lithium niobate at a temperature of $T=120{ }^{0} \mathrm{C}$ calculated from the Sellmeier equation given in Ref. 24. The nonlinear coupling coefficients are the same as the ones used in the previous section.

Figure 13 shows the calculated parametric transfer fidelity $Z$ as a function of the idler carrier wavelength $\left(\lambda_{i}\right)$ for four different pump carrier wavelengths $\left(\lambda_{p}=0.95,1.00,1.05\right.$, and $1.10 \mu \mathrm{m})$ in a $1 \mathrm{~cm}$ long crystal. The tuning was achieved by varying the signal carrier 


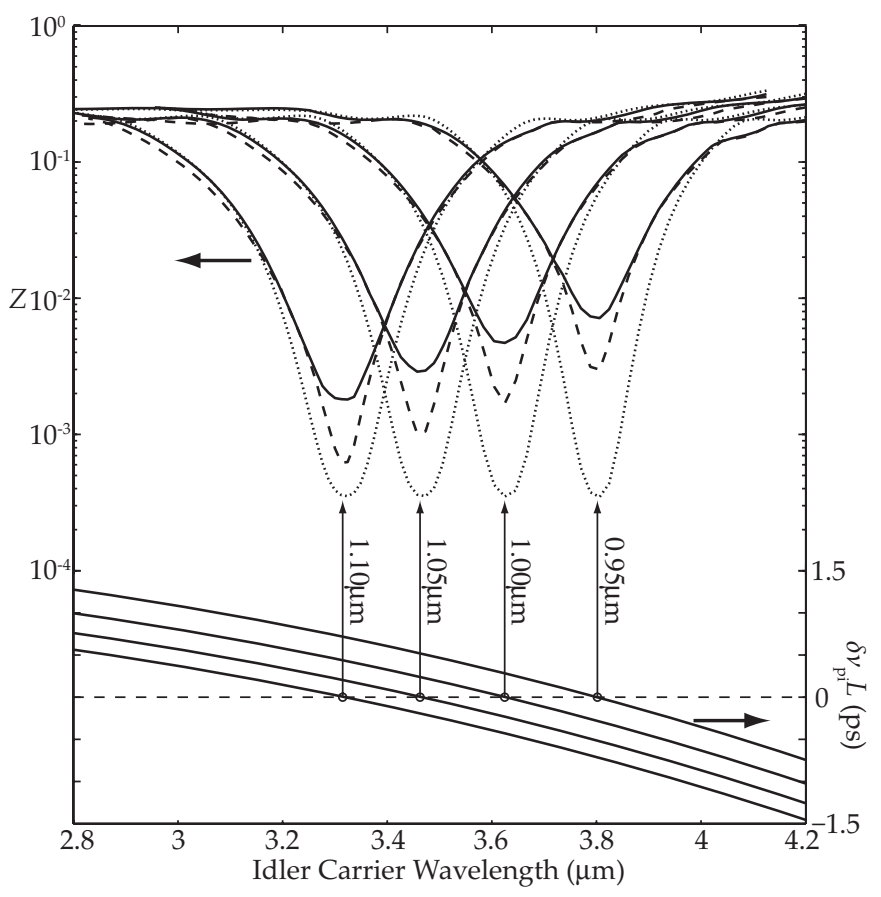

Fig. 13. Parametric transfer fidelity $Z$ (top) and temporal walk-off $\delta \nu_{p i} L$ (bottom) as a function of the idler carrier wavelength $\lambda_{i}$ using different pump carrier wavelength $\lambda_{p}$, as indicated by the numbers, in a $1 \mathrm{~cm}$ long PPLN. The peak intensities for both input pump and signal pulses were $5 \mathrm{MW} / \mathrm{cm}^{2}$. Parametric transfer fidelities for different cases are shown: with GVD (solid curves), with GVD and post-compensation (dashed curves), and without GVD (dotted curves). 
wavelength $\left(\lambda_{s}\right)$, and we assumed that the phase-matching condition is always met, for example, by using different gratings periods, or by changing the temperature. The input pump and signal peak intensities were $I_{p}^{(0)}=I_{s}^{(0)}=5 \mathrm{MW} / \mathrm{cm}^{2}$, resulting in idler peak intensity of $I_{i}^{(0)} \approx 1 \mathrm{MW} / \mathrm{cm}^{2}$. This power level corresponds to low signal amplification, and some pump depletion. Values of $\delta \nu_{p i} L$ as a function of $\lambda_{i}$ for different $\lambda_{p}$ are also plotted in Fig. 13. For different $\lambda_{p}$, there is an idler carrier wavelength at which $Z$ is minimum, which corresponds to zero GVM between the pump and idler pulses. It can be easily observed that as $\lambda_{i}$ varies, $\left|\delta \nu_{p i} L\right|$ rapidly increases, resulting in the narrowing of the spectral filter, and thus limiting the tunability range that can be achieved. Other parts of the MIR spectral regime can be covered, as shown, without reducing the efficiency by using different pump wavelengths. It should be remembered, nonetheless, that in this simulation, we have tried to transfer a relatively large bandwidth $(4.44 \mathrm{THz})$ in a long $(1 \mathrm{~cm})$ PPLN crystal.

In Fig. 13, we plotted calculation results which either include (solid curves), or do not include GVD (dotted curves). The Nelder-Mead algorithm was used on the relative delay $\tau$ to compensate $\delta \nu_{p s}$ and some of the GVD in order to obtain optimal $Z$ in both cases. We also used the Nelder-Mead algorithm to optimize the amount of the post-compensation and the relative delay simultaneously (dashed curves). In general, results without GVD yield a lower $Z$ than the ones with GVD, while the results that use post-compensation fall in between. While the results without GVD show similar behavior for different pump carrier wavelengths, the results with GVD do not show similar behavior. As $\lambda_{p}$ becomes shorter, the transfer fidelity becomes worse, because $\beta_{p}$ becomes larger. This is supported by the fact that the post-compensation is less able to improve the transfer fidelity at shorter $\lambda_{p}$. A similar picture can be obtained for lower input peak intensities.

Utilization of shorter nonlinear crystal can also increase the tuning range. Figure 14 shows the calculated transfer fidelity $Z$ as a function of $\lambda_{i}$ using $\lambda_{p}=1.05 \mu \mathrm{m}$ in three different lengths of PPLN $(L=10,5,2.5 \mathrm{~mm})$. The peak intensities for both input pump and signal pulses were $I_{p}^{(0)}=I_{s}^{(0)}=5,10,20 \mathrm{MW} / \mathrm{cm}^{2}$, respectively. These intensity levels should produce similar output idler peak intensities $I_{i}^{(0)} \approx 1 \mathrm{MW} / \mathrm{cm}^{2}$. Results including GVD, including GVD with post-compensation, and not including GVD, have been plotted. In general, shorter crystal length improves the transfer fidelities, and the post-compensation scheme always improves the results where GVD is included. In a $2.5 \mathrm{~mm}$ long PPLN crystal, results that include GVD (with or without compensation) provide better transfer fidelities than the one that does not include GVD. As explained in Subsection 4.C, in this particular example, the amount of chirp introduced in the material fortuitously improves the limited resolution resulting from the convolution.

These calculations are not only limited to PPLN, but can also be applied to other materials. Chromatic dispersion of different materials results in different sets of wavelengths that satisfy 


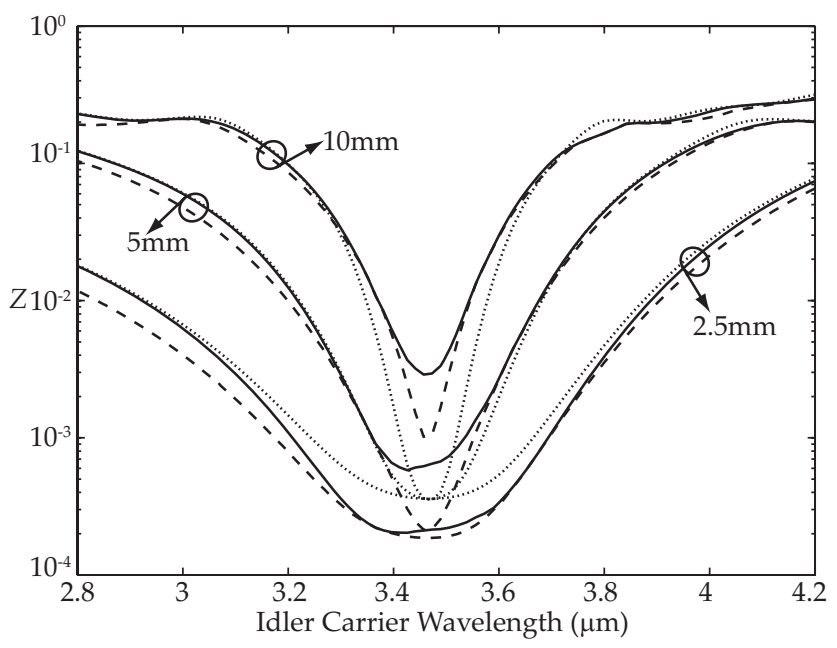

Fig. 14. Parametric transfer fidelity $Z$ (bottom) as a function of the idler carrier wavelength $\lambda_{i}$ using a pump carrier wavelength $\lambda_{p}=1.05 \mu \mathrm{m}$ in three different lengths of PPLN ( $L=10,5,2.5 \mathrm{~mm})$. The peak intensities for both input pump and signal pulses were $I_{p}^{(0)}=I_{s}^{(0)}=5,10,20 \mathrm{MW} / \mathrm{cm}^{2}$, respectively. Parametric transfer fidelities for different cases are shown: with GVD (solid curves), with GVD and post-compensation (dashed curves), and without GVD (dotted curves). 


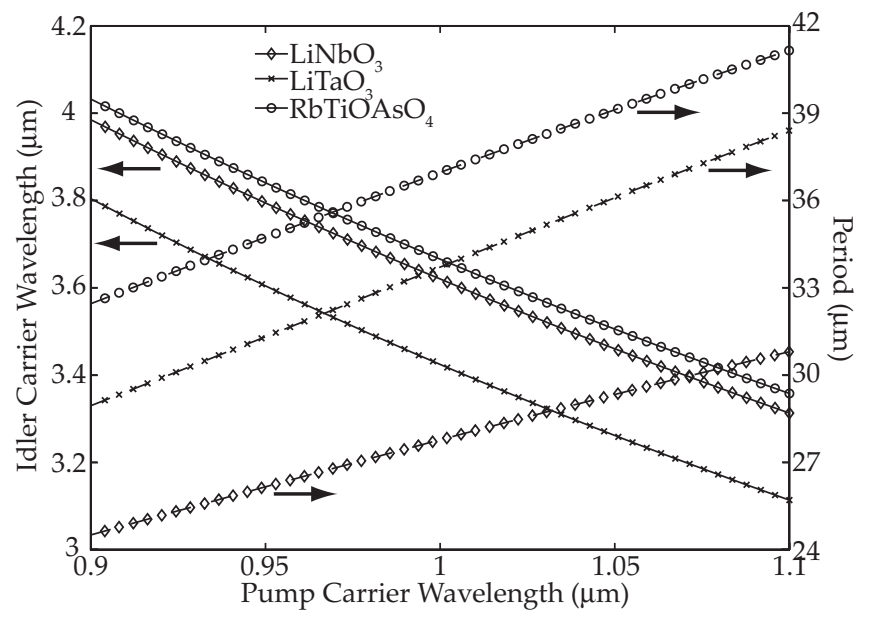

Fig. 15. Idler carrier wavelength $\lambda_{i}$ which has the same group velocity as the pump carrier wavelength, as calculated from the Sellmeier equation for $\mathrm{LiNbO}_{3},{ }^{24} \mathrm{LiTaO}_{3},{ }^{25}$ and $\mathrm{RbTiOAsO}_{4} \cdot{ }^{26}$ The required periods to quasi-phasematch the interaction are also shown in the figure.

$\delta \nu_{p i}=0$. In Fig. 15, we compare the idler carrier wavelengths that satisfy this condition as a function of pump idler carrier wavelengths for $\mathrm{LiNbO}_{3}, \mathrm{LiTaO}_{3}$, and $\mathrm{RbTiOAsO}_{4}$, using the Sellmeier equations given in Refs. 24, 25, and 26, respectively. These materials are commonly used to generate pulses in the MIR regime, and can be engineered for quasi-phase-matching technique. For the same pump carrier wavelengths, $\mathrm{RbTiOAsO}_{4}$ requires a longer idler carrier wavelength to satisfy $\delta \nu_{p i}=0$, followed by $\mathrm{LiNbO}_{3}$, and $\mathrm{LiTaO}_{3}$.

\section{Discussion}

In the previous sections, we have made a comprehensive study of the various effects that can affect the fidelity of the parametric transfer of a shaped NIR pump pulse to the MIR via DFG. We have seen that the parametric transfer is fundamentally limited by the convolution, restricting the resolution of the indirect pulse shaping technique. Therefore, using signal pulses with a narrow spectrum for experimental realizations is imperative. We have addressed the effect of material dispersion, which manifests itself in temporal walk-off and group velocity dispersion. The temporal walk-off between pump and idler pulses restricts the amount of spectral bandwidth one can transfer, while the temporal walk-off between pump and signal pulses shifted their overall temporal overlap. The latter can be compensated by introducing a relative delay, which experimentally can be simply achieved by using a delay line. We have also investigated the effect of the process nonlinearity, i.e. signal amplification 
and pump depletion, on the parametric transfer fidelity. For good fidelity parametric transfer, the input intensities have to be arranged to remain in the regime where pump depletion may happen, but not back conversion. Working in the regime where signal amplification is significant, on the other hand, will always degrade the parametric transfer. Therefore, this limitation of pump and signal intensities to maintain good fidelity transfer will necessarily restrict the intensity of the idler pulses that can be generated. It is worth noting here, that we have also considered contributions of other nonlinear effects in the material, such as selfand cross-phase modulation, but these are found to be negligible under the condition of the calculations presented here.

Several techniques are currently available to overcome the most restrictive factor of parametric transfer, namely the spectral filter effect caused by the temporal walk-off between the pump and idler pulses. One of the most established and successful ones is the use of aperiodically poled material (QPM engineering), which effectively broadens the spectral filter. ${ }^{18}$ Another alternative approach is the critical phase matching scheme involving spatial walk-off with pulse-front tilt. ${ }^{27}$

Operation at different, in this case longer, wavelengths could be achieved via a tandem configuration. In such a configuration, two or more DFG processes in different materials may take place. The idler pulses produced in one DFG are used as the pump pulses of another DFG, producing longer wavelength idler pulses. Interesting materials for this configuration includes CdSe, ${ }^{28}$ and orientation-patterned GaAs. ${ }^{29}$

It should be noted that coherent control applications are not strictly reliant on achieving good fidelity transfer, but rather just require the production of a particular MIR shaped pulse via a closed-loop learning technique, whatever the input pump pulse shape. We can view this approach as an application of amplitude and phase corrections to the shaped pump pulse to mitigate some factors degrading the parametric transfer fidelity, such as GVD, back conversion, and signal amplification. Using this approach, intense idler pulses with a particular shape could in principle be produced. Further studies are necessary to verify the capabilities and limitations of this approach. However, there are factors degrading the parametric transfer fidelity that can not be mitigated. The main factor would be the convolution itself, which limits the resolution, and the temporal walk-off between the pump and the idler pulses, which limits the spectral bandwidth that can be transferred. These problems need to be addressed, as the learning techniques used will not be able to overcome them.

Since one can argue that the generation of the idler pulse in an OPO device is a result of a DFG process, albeit the resonating signal pulse being built up from noise, we can expect that results in this paper can be qualitatively applied to it. ${ }^{30}$ For example, the necessary narrow spectrum signal pulse could be provided by restricting the spectral bandwidth of 
the resonating signal pulse using an intra-cavity spectral filter. Such a spectral filter would increase the effective loss of the cavity, and thus the required threshold value. We expect that the relative delay between the pump and signal pulses to compensate their temporal walk-off can be mimicked by adjusting the cavity length. Furthermore, the use of an output coupling for the signal pulses should not be needed, resulting in a reduced cavity losses and the amount of necessary signal amplification. Theoretical and numerical analysis for synchronously-pumped OPO (SPOPO) to clarify these issues will be the subject of our future work.

\section{Summary}

We have theoretically and numerically analyzed the main factors affecting the fidelity of the parametric transfer of a shaped NIR pulse to the MIR via a DFG process for indirect pulse shaping techniques beyond convolution. These factors include material dispersion (GVM and GVD), and process nonlinearity (signal amplification and pump depletion). We showed that a good fidelity transfer of a broad-bandwidth pulse can be efficiently achieved with a reasonable wavelength tunability, by careful design of the experimental configuration.

\section{Acknowledgment}

This work was supported by EPSRC Instrument Grant EP/C009479/1. Hazel S. S. Hung acknowledges the support of an EPSRC studentship. Numerical simulations in this work were carried out using the IRIDIS computational facility, ${ }^{31}$ at the University of Southampton.

J. Prawiharjo's e-mail address is jep@orc.soton.ac.uk

\section{References}

1. S. Woutersen, U. Emmerichs, and H. J. Bakker, "Femtosecond Mid-IR Pump-Probe Spectroscopy of Liquid Water: Evidence for a Two-Component Structure," Science 278, 658 (1997).

2. R. A. Kaindl, S. Lutgen, M. Woerner, T. Elsaesser, B. Nottelmann, V. M. Axt, T. Kuhn, A. Hase, and H. Künzel, "Ultrafast Dephasing of Coherent Intersubband Polarizations in a Quasi-Two-Dimensional Electron Plasma," Physical Review Letters 80, 3575 (1998).

3. R. A. Kaindl, M. Woerner, T. Elsaesser, D. C. Smith, J. F. Ryan, G. A. Farnan, M. P. McCurry, and D. G. Walmsley, "Ultrafast Mid-Infrared Response of $\mathrm{YBa}_{2} \mathrm{Cu}_{3} \mathrm{O}_{7-\delta}$," Science 287, 470 (2000).

4. H. Rabitz, R. de Vivie-Riedle, M. Motzkus, and K. Kompa, "Whither the Future of Controlling Quantum Phenomena?" Science 288, 824 (2000).

5. R. N. Zare, "Laser Control of Chemical Reactions," Science 279, 1875 (1998). 
6. V. D. Kleiman, S. M. Arrivo, J. S. Melinger, and E. J. Heilweil, "Controlling CondensedPhase Vibrational Excitation with Tailored Infrared Pulses," Chemical Physics 233, 207 (1998).

7. L. Windhorn, T. Witte, J. S. Yston, D. Proch, M. Motzkus, K. L. Kompa, and W. Fuß, "Molecular Dissociation by Mid-IR Femtosecond Pulses," Chemical Physics Letters 357, 85 (2002).

8. A. M. Weiner, D. E. Leaird, J. S. Patel, and I. J. R. Wullert, "Programmable Shaping of Femtosecond Optical Pulses by Use of 128-Element Liquid Crystal Phase Modulator." IEEE Journal of Quantum Electronics 28, 908 (1992).

9. E. Zeek, K. Maginnis, S. Backus, Ulrussek, M. Murnane, G. Mourou, H. Kapteyn, and G. Vdovin, "Pulse Compression by Use of Deformable Mirrors," Optics Letters 24, 493 (1999).

10. F. Verluise, V. Laude, Z. Cheng, C. Spielmann, and P. Tournois, "Amplitude and Phase Control of Ultrashort Pulses by Use of an Acousto-Optic Programmable Dispersive Filter: Pulse Compression and Shaping," Optics Letters 25, 575 (2000).

11. S. H. Shim, D. B. Strasfeld, E. C. Fulmer, and M. T. Zanni, "Femtosecond Pulse Shaping Directly in the Mid-IR Using Acousto-Optic Modulation," Optics Letters 31, 838 (2006).

12. F. Eickemeyer, R. A. Kaindl, M. Woerner, T. Elsaesser, and A. M. Weiner, "Controlled Shaping of Ultrashort Electric Field Transients in the Mid-Infrared Spectral Range," Optics Letters 25, 1472 (2000).

13. N. Belabas, J. P. Likforman, L. Canioni, B. Bousquet, and M. Joffre, "Coherent Broadband Pulse Shaping in the Mid Infrared," Optics Letters 26, 743 (2001).

14. H. S. Tan, W. Schreiber, and W. S. Warren, "High-Resolution Indirect Pulse Shaping by Parametric Transfer," Optics Letters 27, 439 (2002).

15. T. Witte, D. Zeidler, D. Proch, K. L. Kompa, and M. Motzkus, "Programmable Amplitude- and Phase-Modulated Femtosecond Laser Pulses in the Mid-Infrared," Optics Letters 27, 131-133 (2002).

16. T. Witte, K. L. Kompa, and M. Motzkus, "Femtosecond Pulse Shaping in the Mid Infrared by Difference-Frequency Mixing," Applied Physics B 76, 467 (2003).

17. W. S. Tan and W. S. Warren, "Mid Infrared Pulse Shaping by Optical Parametric Amplification and Its Application to Optical Free Induction Decay Measurement," Optics Express 11, 1021 (2003).

18. G. Imeshev, M. A. Arbore, M. M. Fejer, A. Galvanauskas, M. Fermann, and D. Harter, "Ultrashort-Pulse Second-Harmonic Generation with Longitudinally Nonuniform QuasiPhase-Matching Gratings: Pulse Compression and Shaping," Journal of the Optical Society of America B 17, 304 (2000).

19. G. Imeshev, M. M. Fejer, A. Galvanauskas, and D. Harter, "Pulse Shaping by Difference- 
Frequency Mixing with Quasi-Phase-Matching Gratings," Journal of the Optical Society of America B 18, 534 (2001).

20. R. Trebino, K. W. DeLong, D. N. Fittinghoff, J. N. Sweetser, and M. A. Krumbügel, "Measuring Ultrashort Laser Pulses in the Time-Frequency Domain Using FrequencyResolved Optical Gating," Reviews of Scientific Instruments 68, 3277 (1997).

21. J. Prawiharjo, K. Gallo, N. G. R. Broderick, and D. J. Richardson, "Frequency-Resolved Optical Gating in the $1.55 \mu \mathrm{m}$ Band Via Cascaded $\chi^{(2)}$ Processes," Journal of the Optical Society of America B 22, 1985 (2005).

22. G. P. Agrawal, Nonlinear Fiber Optics (Academic Press, London, 2001), third edn.

23. J. C. Lagarias, J. A. Reeds, M. H. Wright, and P. E. Wright, "Convergence Properties of the Nelder-Mead Simplex Method in Low Dimensions," SIAM Journal of Optimizations 9, 112 (1998).

24. D. Jundt, "Temperature-Dependent Sellmeier Equation for the Index of Refraction, $n_{e}$, in Congruent Lithium Niobate," Optics Letters 22, 1553 (1997).

25. K. S. Abedin and H. Ito, "Temperature-Dependent Dispersion Relation of Ferroelectric Lithium Tantalate," Journal of Applied Physics 80, 6561 (1996).

26. K. Kato, E. Takaoka, and N. Umemura, "Thermo-Optic Dispersion Formula for $\mathrm{RbTiOAsO}_{4}$," Japanese Journal of Applied Physics 42, 6420 (2003).

27. A. M. Schober, M. Carbonneau-Lefort, and M. M. Fejer, "Broadband Quasi-PhaseMatched Second-Harmonic Generation of Ultrashort Optical Pulses with Spectral Angular Dispersion," Journal of the Optical Society of America B 22, 1699 (2005).

28. M. A. Watson, M. V. O'Connor, D. P. Shepherd, and D. C. Hanna, "Synchronously Pumped CdSe Optical Parametric Oscillator in the $9-10 \mu \mathrm{m}$ Region," Optics Letters 28, 1957 (2003).

29. P. S. Kuo, K. L. Vodopyanov, M. M. Fejer, D. M. Simanovskii, X. Yu, J. S. Harris, D. Bliss, and D. Weyburne, "Optical Parametric Generation of a Mid-Infrared Continuum in Orientation-Patterned GaAs," Optics Letters 31, 71 (2006).

30. H. S. S. Hung, N. A. Naz, J. Prawiharjo, D. P. Shepherd, and D. C. Hanna, "Parametric Transfer in a Synchronously Pumped Optical Parametric Oscillator," Presented at CLEO/QELS 2006 Long Beach, California 21-25 May 2006.

31. http://www.iss.soton.ac.uk/research/iridis. 